\title{
Guiding binocular saccades during reading: aTMS study of the PPC
}

\author{
Marine Vernet ${ }^{1,2 *}$, Qing Yang ${ }^{1}$ and Zoï Kapoula \\ IRIS Laboratory, CNRS, FRE 3154 and Assistance Publique - Hôpitaux de Paris, Paris, France \\ 2 University of Pierre and Marie Curie (Paris 6), Paris, France
}

Edited by:

Alvaro Pascual-Leone, Beth Israel Deaconess Medical Center/Harvard Medical School, USA

\section{Reviewed by:}

Phillip R. Zoladz, Ohio Northern

University, USA

Paul M. Nealen, Indiana University of

Pennsylvania, USA

*Correspondence:

Marine Vernet, IRIS Laboratory, CNRS FRE 3154, Service d'ophtalmologie, Hôpital Européen Georges Pompidou, 20 rue Leblanc, 75015 Paris, France. e-mail:marine.vernet@gmail.com
Reading is an activity based on complex sequences of binocular saccades and fixations. During saccades, the eyes do not move together perfectly: saccades could end with a misalignment, compromising fused vision. During fixations, small disconjugate drift can partly reduce this misalignment. We hypothesized that maintaining eye alignment during reading involves active monitoring from posterior parietal cortex (PPC); this goes against traditional views considering only downstream binocular control. Nine young adults read a text; transcranial magnetic stimulation (TMS) was applied over the PPC every $5 \pm 0.2 \mathrm{~s}$. Eye movements were recorded binocularly with Eyelink II. Stimulation had three major effects: (1) disturbance of eye alignment during fixation; (2) increase of saccade disconjugacy leading to eye misalignment; (3) decrease of eye alignment reduction during fixation drift. The effects depend on the side; the right PPC was more involved in maintaining alignment over the motor sequence. Thus, the PPC is actively involved in the control of binocular eye alignment during reading, allowing clear vision. Cortical activation during reading is related to linguistic processes and motor control per se. The study might be of interest for the understanding of deficits of binocular coordination, encountered in several populations, e.g., in children with dyslexia.

Keywords: reading, binocular coordination, transcranial magnetic stimulation, posterior parietal cortex, saccade, fixation

\section{INTRODUCTION}

Reading involves saccades and fixations. During saccades, both eyes move quickly in the same direction by the same amplitude. Binocular motor coordination helps to obtain fused vision during fixations, by allowing the vergence angle (angle between the lines of sight of the two eyes) to be stable and appropriate for the distance of fixation. Yet, the binocular motor coordination is not perfect. When saccading between isolated targets, the abducting eye is generally faster than the adducting eye, causing a divergent disconjugacy (lines of sight are crossing further away); this tendency is reversed toward the saccade end. The residual misalignment between the eyes can be further reduced via a postsaccadic fixation drift (Collewijn et al., 1988). Less often, saccades could end with a convergent (lines of sight crossing closer) or null (lines of sight crossing at the same distance) vergence error (Yang and Kapoula, 2003). This transient saccade disconjugacy is traditionally believed to reflect peripheral asymmetries (premotor circuits and visco-elastic properties of the ocular muscles, see, e.g., Collewijn et al., 1988; Zee et al., 1992). During reading, the coordination is also not perfect. The eyes do not always fixate the same character (Blythe et al., 2006; Liversedge et al., 2006a; Kirkby et al., 2008; Nuthmann and Kliegl, 2009), but the disconjugacy remains small (Vernet and Kapoula, 2009). The aim of the present study is to investigate the cortical basis of such $3 \mathrm{D}$ control during reading.

Transcranial magnetic stimulation (TMS) allows a reversible perturbation of a cortical area. Neural substrates can be investigated directly in healthy humans (Jahanshahi and Rothwell, 2000).
To our knowledge, only one study (Leff et al., 2001) used TMS to investigate the neurophysiology of eye movements during reading. A train of pulses, i.e., repetitive TMS (rTMS), over the left posterior parietal cortex (PPC) slowed total reading speeds; rTMS over the right frontal eye field (FEF) slowed the time to make the first saccade. The left PPC controls the "on-line" maintenance of a sensorimotor plan to read; the right FEF is responsible for the preparation of this plan.

The PPC is a crossroad of sensorimotor signals, encoded in 3D coordinates (Sakata et al., 1980; Gnadt and Mays, 1995; Colby et al., 1996). Concerning the temporal control of 3D eye movements, single-pulse TMS on the PPC delayed the latency of saccades and vergence (Kapoula et al., 2001; Yang and Kapoula, 2004). Concerning the binocular coordination, single-pulse TMS on the PPC increased disconjugacy during $10^{\circ}$ saccades (Vernet et al., 2008).

Saccades during reading are smaller than saccades toward single targets generally studied. They belong to a complex sequence, done in parallel with words identification and linguistic processes. On the other hand, reading is an overlearned motor task. Grosbras et al. (2001) showed that newly learned and familiar sequences of saccades activated a common fronto-parietal network, with higher and larger activation for new sequences. However, familiar sequences recruited two specific areas: one in the right medial temporo-occipital cortex, the second in the upper (parietal) branch of the right parieto-occipital sulcus, extending medially toward the most posterior part of the cingulate gyrus. The latter might be involved into the computation of extraretinal target coordinates and associated eye movements. 
In the present study, we examined the effect of single-pulse TMS over the PPC on temporal reading parameters, but also on the binocular motor control. Considering reading movements as learned sequences, we expected that the PPC, and particularly the right PPC, would be highly active during reading and that its stimulation would affect both time parameters and binocular coordination.

\section{MATERIALS AND METHODS SUBJECTS}

Nine adult subjects (six women and three men) were tested. Their ages ranged from 19.3 to 41.4 years (mean, 28.2 \pm 8.7 years). Seven were native French speakers, one was native Chinese speaker and one was native German speaker, both of them fluent in English. All were normal readers and had at least 2 years of study in university. As the oculomotor behavior was not different for different native language, all data were examined together. All subjects were healthy and did not have any neurologic, neuro-otologic, or ophthalmologic symptoms. They had normal or corrected-to-normal vision. Binocular vision was assessed with the TITMUS test of stereoacuity. All individual scores were normal (40 s of arc or better). Each participant gave informed consent to participate in the experiment. This investigation was approved by the local ethics committee and was consistent with the Declaration of Helsinki.

\section{TRANSCRANIAL MAGNETIC STIMULATION}

Single-pulse stimulation was applied with a magnetic stimulator (model 200, MagStim, UK). Maximum stimulator output was $2.2 \mathrm{~T}$; the coil had a figure-of-eight (each wing $70 \mathrm{~mm}$ diameter). The cortical area for which stimulation provoked the most visible jerks of contralateral hand muscles was defined as the motor hand area. Then the intensity of the stimulator was adjusted until it reached a value for which visible jerks of contralateral hand muscles occurred on $50 \%$ of the trials. For our group of subjects, the motor threshold ranked from 40 to $60 \%$ of total stimulator output. The left or right $\mathrm{PPC}$ was stimulated by placing the coil $3 \mathrm{~cm}$ posteriorly and $3 \mathrm{~cm}$ laterally to the vertex, tangentially to the skull, with its handle oriented backward and $45^{\circ}$ exterior. The left and right targeted stimulation sites were on average $2 \mathrm{~cm}$ from $\mathrm{P} 3$ and $\mathrm{P} 4$ respectively, as defined in the 10-20 EEG system. During PPC stimulation, the intensity was set $20 \%$ above motor threshold. For some subjects, this intensity caused blink; the intensity was consequently decreased but remained 10\% above motor threshold. Thus, for our group of subjects, the intensity for PPC stimulation was between 45 and $70 \%$ of total stimulator output.

During reading, single-pulse stimulation was delivered every $5 \pm 0.2 \mathrm{~s}$. The occurrence of a stimulation pulse was random relative to the eye position in the text. Single-pulse stimulation is believed to deteriorate ongoing neural processes and perturbation is estimated to last up to $250 \mathrm{~ms}$ after stimulation (Jahanshahi and Rothwell, 2000). Here we hypothesized that PPC could be involved in active online processes for maintaining binocular coordination during entire sequences of saccades and fixations; thus, TMS effects will be examined up to $600 \mathrm{~ms}$ after a pulse of stimulation.

For the condition without TMS, stimulation was also delivered, but the coil was placed $30 \mathrm{~cm}$ over the head of the subject, oriented toward the ceiling and a second coil, unlinked to the magnetic stimulator, was placed over the subject head (same auditory and somatosensory cues as during real stimulation, see, e.g., Kapoula et al., 2004, 2005; Yang and Kapoula, 2004).

\section{EYE MOVEMENT RECORDING}

Eye movements were recorded binocularly (EyeLink II, SR Research Ltd., Canada). Each channel was sampled at $250 \mathrm{~Hz}$. The system had an accuracy (absolute position error) of $0.5^{\circ}$ and a resolution (smallest variation of position detectable) of $0.025^{\circ}$. Crosstalk compensation was performed with the EyeLink calibration (nine points), with a non-linear function (Stampe, 1993). A second calibration (described below) followed at the beginning of each block.

\section{PROCEDURE}

The subject was comfortably seated, the head stabilized with a chin rest, and viewed binocularly the $\mathrm{PC}$ screen placed at $40 \mathrm{~cm}$. At the beginning of each block, a calibration was run: a target jumped between five positions on the screen, at the centre and at $\pm 11.6^{\circ}$ horizontally and $\pm 12.4^{\circ}$ vertically; the target remained at each position during $1 \mathrm{~s}$. The subject was asked to follow accurately the target. In this study, we do not measured absolute fixation disparity, but disconjugacy, i.e., change in disparity, during the saccades and during the fixations. Measures of disconjugacy are statistically similar after monocular or binocular calibration, even in children with strabismus (Bucci et al., 2002).

During the reading task, a text in French appeared, "L'alouette" (279 words), containing non-frequent words, commonly used in France for evaluation of reading capacity. For the two non-native French speakers, a length-equivalent English extract from the "Discourse on Method" by Descartes appeared. This text in formal language is commonly used in French clinical ophthalmology consultations for evaluation of visual acuity. Both texts contain a high number of non-frequent words and the average reader cannot neither use anticipation nor learn the text with few presentations.

The texts were written in courier font, each letter about $0.45^{\circ}$ of angular size. Three text panels of $\pm 9.9^{\circ}$ width and $\pm 8.9^{\circ}$ height were presented on the screen for $30 \mathrm{~s}$ each, covering the total text. The subjects should read silently when it appears. Before the text appearance they had to fixate a target at the lower left end of the screen; they were asked to fixate the same target after reading completion. The reading time comprised the time between the first saccade toward the text and the saccade back toward the target. The subjects read the text one time for each TMS condition (left PPC, right PPC, no-TMS), in a randomized order. In order to check that they actually read the text, they were each time asked to briefly comment on it. As will be shown in the Section "Results", the total reading time was the same for the three reading. The randomization of the TMS condition across subjects further prevents to confound TMS effect with effects arising from reading several times the same text.

\section{DATA ANALYSIS}

Calibration was run on the eye position signals with second order polynomial functions. We then derived the horizontal and vertical conjugate signal (mean of the two eyes position) and the horizontal disconjugate signal (left-right eye). 
Saccades with amplitude larger than $1^{\circ}$ were examined. The onset (respectively, the offset) of saccades was defined as the time when the eye velocity of the conjugate signal exceeded (respectively, dropped below) 10\% of the maximum velocity. The end of the first fixation period of $160 \mathrm{~ms}$ following the saccade was marked for fixation longer than $160 \mathrm{~ms}$. Indeed, the time constant of physiological drift related to saccade control, i.e., the time constant describing the pulse-slide-step activity recorded in abducens neurons, is below $160 \mathrm{~ms}$ (Leigh and Zee, 2006). The fixations shorter than $160 \mathrm{~ms}$ were not considered in the analysis. Moreover, the few fixations containing conjugate movements superior to $1^{\circ}$ and/or small saccades (between $0.5^{\circ}$ and $1^{\circ}$ ) were also discarded. Thus, the number of fixation periods of $160 \mathrm{~ms}$ was slightly lower than the number of saccades (Table $\mathbf{1}$ ).

In the following, the term "saccades" refers to rightward saccades. The term "regressive saccades" refers to leftward saccades within one line of text. We measured the amplitude, duration, and disconjugacy of the saccades and of the post-saccadic drifts during the first $160 \mathrm{~ms}$ of fixation. The disconjugacy is the modification of the disconjugate signal, or vergence signal, during the saccade or during the fixation. We also calculated the percentage of saccades or fixations with divergent (negative) disconjugacy and then the average absolute value of disconjugacy.

The effects of TMS on reading oculomotor parameters were assessed in a time-window of $600 \mathrm{~ms}$ starting at the TMS delivery. Such time-window was sufficiently large to include a sufficient number of saccades $(23 \pm 5)$ and fixations lasting at least $160 \mathrm{~ms}$ $(17 \pm 5)$ per subject and per TMS condition. Even if the effects of single-pulse TMS are limited in time, we hypothesized that the PPC might be involved in the binocular control of entire saccade sequences and that consequently disconjugacy might be disturbed up to $600 \mathrm{~ms}$ after TMS of the PPC.

One-way repeated-measures ANOVA was applied on each parameter to test the effect of TMS. Least significant difference (LSD) test of Fisher was used for post hoc two-by-two comparisons. In addition, when the effects measured were statistically significant, Cohen's $d$ values were calculated to assess the strength of the effect independently of the number of subjects considered. Nonparametric Friedman test was used for testing the effect of TMS on the percentages of saccades or fixations with divergent disconjugacy. Finally, Pearson correlation coefficients were calculated to test for the existence of a relation between saccade disconjugacy and drift disconjugacy.

\section{RESULTS \\ GENERAL READING CHARACTERISTICS}

Table 1 indicates the average number of rightward saccades and of regressive saccades (leftward saccades within a line of text; saccades of return to the next line are not taken into account) per line and the total reading duration. On average, a subject made 5.5 rightward saccades and 0.6 regressive saccades per line. These numbers were slightly higher with TMS over left and right PPC, but this effect was not statistically significant (all the $p>0.05$ ). TMS had no effect on the total reading time (all the $p>0.05$ ).
Table 1 | General reading characteristics: number of saccades, number of regressive saccades (within a line of text, saccades of return to the next line excluded) and total reading duration (mean \pm SD).

\begin{tabular}{llll}
\hline & $\begin{array}{l}\text { N rightward } \\
\text { sac./lines }\end{array}$ & $\begin{array}{l}\text { N regressive } \\
\text { sac./lines }\end{array}$ & $\begin{array}{l}\text { Total reading } \\
\text { duration }(\mathbf{s})\end{array}$ \\
\hline No-TMS & $5.5 \pm 0.9$ & $0.6 \pm 0.5$ & $78 \pm 17$ \\
TMS of LPPC & $5.7 \pm 0.8$ & $0.7 \pm 0.6$ & $80 \pm 16$ \\
TMS of RPPC & $5.7 \pm 0.8$ & $0.7 \pm 0.6$ & $79 \pm 15$ \\
\hline
\end{tabular}

\section{QUALITATIVE DESCRIPTION OF THE EFFECT OF TMS ON CONJUGATE AND DISCONJUGATE COMPONENTS}

Figure 1 shows samples of conjugate eye position and disconjugacy traces during $1100 \mathrm{~ms}$, corresponding approximately to the reading of one line. Inspection of conjugate traces showed no effect of TMS on conjugate aspect of saccades and fixations, neither on saccade amplitude, nor on conjugate drift. Some disturbance after stimulation occasionally occurred (see subject s6, TMS of the left PPC), but such disturbance was rare and TMS had no statistically significant effect on any conjugate parameters (amplitude, duration, and velocity, all the $p>0.05$ ).

Inspection of disconjugate traces showed that for each saccade in the no-TMS trials, there was a transient disconjugacy that modified the vergence angle; very soon after the end of the saccade, the vergence angle returned approximately to $8.6^{\circ}$, which corresponds to the viewing distance for an inter-ocular distance of $60 \mathrm{~mm}$. This behavior was disturbed by the left PPC and right PPC stimulation: saccade disconjugacy increased and vergence angle remained instable.

\section{DURATION, AMPLITUDE, AND DISCONJUGACY OF SACCADES AND OF POST-SACCADIC DRIFT}

Quantitatively, Figure 2 indicates duration, amplitude, disconjugacy, and percentage of divergent disconjugacy of saccades and fixations.

Figure 2A shows the duration of saccades and fixations for the different TMS conditions. On average, the saccade duration and fixation duration were $34 \pm 4 \mathrm{~ms}$ and $279 \pm 98 \mathrm{~ms}$, respectively. Saccade duration and fixation duration were not significantly modified by TMS ( $p>0.05)$.

Figure 2B shows the amplitude of saccades and fixations for the different TMS conditions. On average, the amplitude of rightward reading saccades was $3.2^{\circ} \pm 0.6^{\circ}$, the absolute value of the conjugate drift was $0.17^{\circ} \pm 0.05^{\circ}$ during the first $160 \mathrm{~ms}$ of fixation. Conjugate saccade amplitude and conjugate fixation drift were not modified by TMS $(p>0.05)$.

Figure 2C shows the percentage of saccades with divergent disconjugacy and the percentage of fixation with divergent disconjugacy for the different TMS conditions. The disconjugacy was mainly divergent during the saccade and mainly convergent during the first $160 \mathrm{~ms}$ of fixation. TMS had no effect on the percentage of saccades or fixations with divergent disconjugacy (Friedman test, all the $p>0.05)$. These percentages were highly idiosyncratic.

On the contrary to previous parameters, TMS of the PPC had significant effect on disconjugacy. Figure 2D shows disconjugacy of saccades and fixations for the different TMS conditions. The 


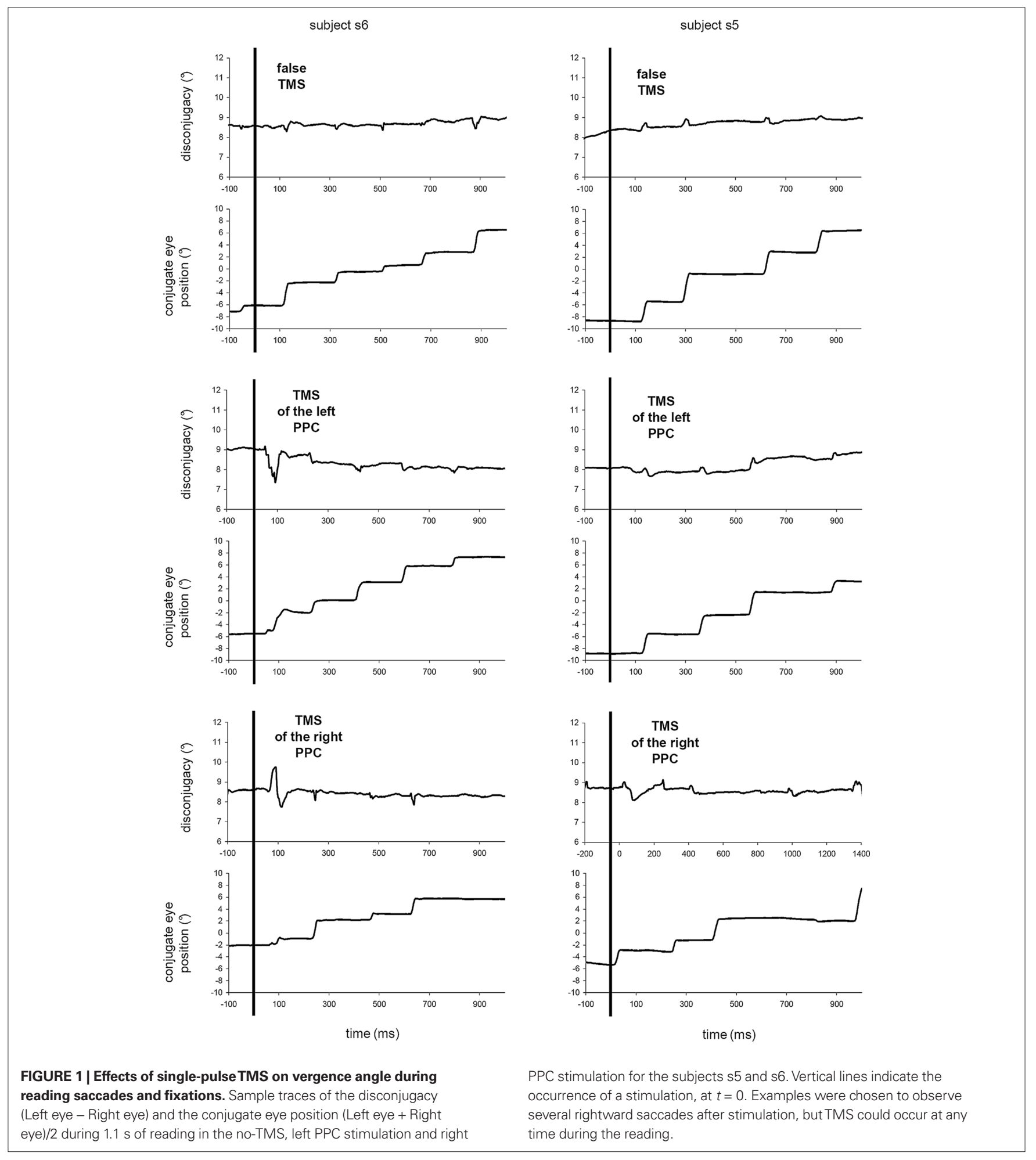

ANOVA showed that TMS had a significant effect on saccades disconjugacy $[F(2,16)=5.56 ; p<0.05]$. Post hoc tests showed that TMS of the right PPC increased significantly saccade disconjugacy (from $0.17^{\circ} \pm 0.05^{\circ}$ to $0.36^{\circ} \pm 0.16^{\circ} ; p<0.01$ ). The Cohen's $d$ value (mean for no-TMS - mean for right PPC TMS/pooled SD) was superior to 1.6, indicating that the effect was strong despite the

number of subjects. The increase of saccade disconjugacy after TMS of the left PPC (from $0.17^{\circ} \pm 0.05^{\circ}$ to $0.26^{\circ} \pm 0.13^{\circ}$ ) was not significant $(p>0.05)$.

Similarly, the ANOVA showed that TMS had a significant effect on drift disconjugacy $[F(2,16)=3.71 ; p<0.05]$. Post hoctests showed that TMS of the right PPC increased significantly drift disconjugacy 
A

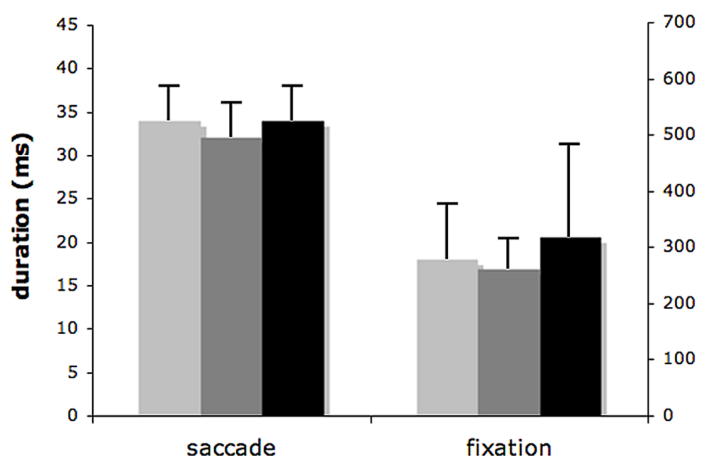

C

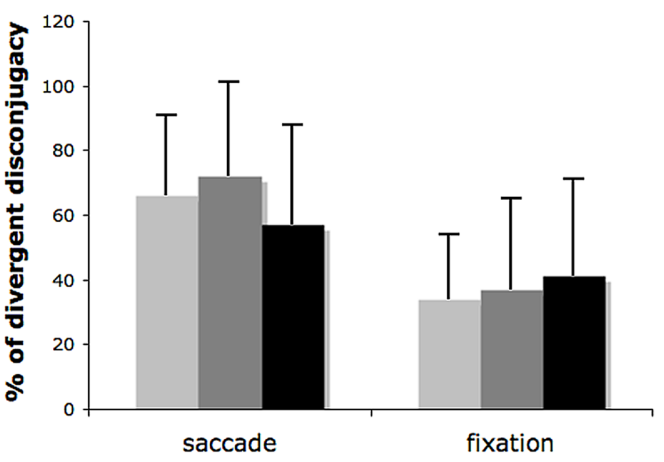

FIGURE 2 | Effects of single-pulse TMS on saccades and fixations. Means and SD of: (A) saccade and fixation duration; (B) saccade and fixation amplitude (in absolute value); (C) percentage of saccade with divergent disconjugacy and

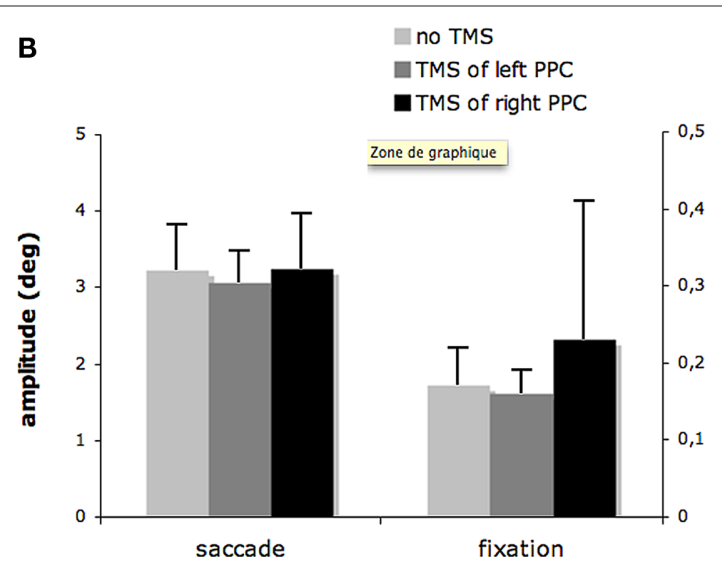

D

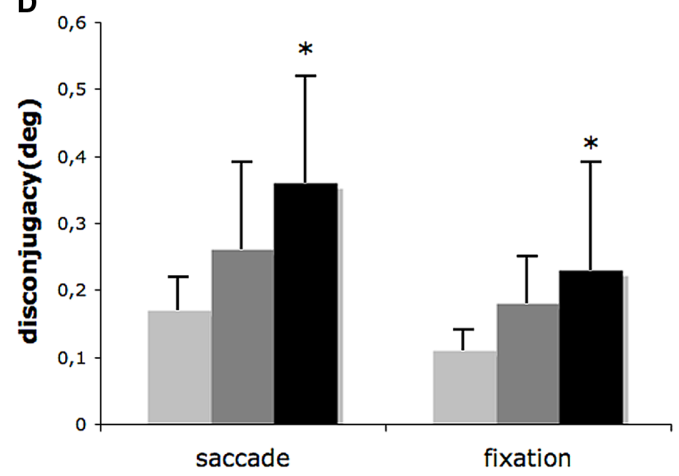

of fixation with divergent disconjugacy; (D) saccade and fixation disconjugacy (in absolute value). Asterisks indicate statistically significant effect of TMS. Note the different scales for saccades and fixations values in (A) and (B). (from $0.11^{\circ} \pm 0.03^{\circ}$ to $0.23^{\circ} \pm 0.16^{\circ} ; p<0.05$ ). The Cohen's $d$ value was superior to 1.0 , indicating that the effect was large despite the number of subjects. The increase of fixation disconjugacy after TMS of the left PPC (from $0.11^{\circ} \pm 0.03^{\circ}$ to $0.18^{\circ} \pm 0.07^{\circ}$ ) was not significant $(p>0.05)$.

\section{CORRELATION BETWEEN SACCADE DISCONJUGACY AND DRIFT DISCONJUGACY}

Figure 3 illustrates for all subjects the relationship between saccade disconjugacy and fixation disconjugacy in the different TMS conditions and also illustrates the increase of saccade disconjugacy and drift disconjugacy with TMS. The correlation between saccade disconjugacy was significant for all TMS condition (all $p<0.001$ ). The correlation coefficient was nevertheless higher without TMS $(r=-0.59)$ than with TMS of the left PPC $(r=-0.31)$ or than with TMS of the right PPC $(r=-0.39)$. Thus, in the no-TMS condition, drift disconjugacy efficiently compensated for saccade disconjugacy. TMS of the left or right PPC disturbed the relationship between saccade disconjugacy and drift disconjugacy. Consequently, the drift disconjugacy is less efficient in reestablishing eye alignment, lost because of prior saccade disconjugacy. Saccade disconjugacy and drift disconjugacy should be considered together in order to better estimate the binocular coordination during reading.

\section{DISCUSSION}

\section{TMS DISTURBED FIXATION DURATION}

Single-pulse TMS applied over the PPC is known to increase the latency of visually guided saccades, i.e., the time between the target onset and the beginning of the saccade, according the saccade direction, the hemisphere stimulated and the timing of stimulation (see, e.g., Kapoula et al., 2001; Yang and Kapoula, 2004). During reading, fixation duration can reflect the saccade preparation, together with several other processes (e.g., identification of the fixated word).

Leff et al. (2001) measured the effect of TMS over the PPC on temporal parameters during reading; the subjects had to read pseudo-sentences made of five unassociated words in a horizontal array. In one of the experiments, rTMS was applied on the left or right PPC. A train of 15 pulses at $10 \mathrm{~Hz}$ was applied, starting at the apparition of the pseudo-sentence and lasting $1500 \mathrm{~ms}$. Left, but not right, PPC stimulation, increased the reading time.

In the present study, the subjects read a text. Similarly to the previous cited study, each word was difficult to predict and remember. The stimulation, applied every $5 \pm 0.2 \mathrm{~s}$, started shortly before the apparition of the text and lasted during all the text reading. No significant effect on the duration of the fixations occurring up to $600 \mathrm{~ms}$ was measured. The lack of significant effect could mean that, contrary to isolated saccades latency, reading saccades latency is more robust to single-pulse TMS disturbance and that contrary to 

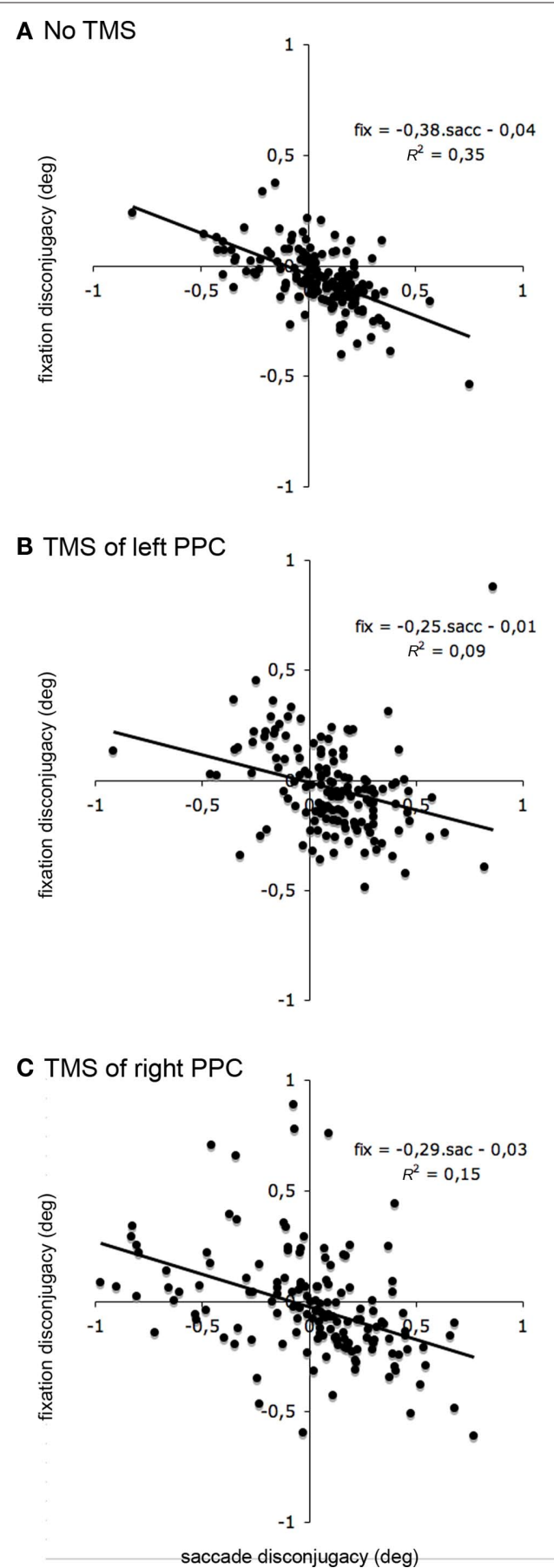

FIGURE 3 | Effects of single-pulse TMS on the link between saccade disconjugacy and fixation drift disconjugacy. Drift disconjugacy plotted against saccade disconjugacy for the saccade occurring in the $600 \mathrm{~ms}$ window, in the no-TMS, left PPC stimulation and right PPC stimulation. Note that outliers have been removed before analysis.

rTMS, single-pulse TMS failed to increase reading saccade latency. Alternatively, and more probably, each single-pulse of TMS might have delayed the latency of the first next saccade, but the effect was no more visible when considering the first few saccades occurring in the entire $600 \mathrm{~ms}$ time-window after TMS pulse.

\section{TMS DISTURBED THE BINOCULAR COORDINATION OF READING SACCADES}

Transcranial magnetic stimulation of the right PPC during reading increased disconjugacy of saccades occurring up to $600 \mathrm{~ms}$ after stimulation. This is consistent with the previous study made on saccades to single targets, where TMS of the right PPC increased the disconjugacy of rightward and downward saccades and TMS of the left PPC increased the disconjugacy of downward saccades only (Vernet et al., 2008). Right and left PPC are differently specialized for the control of binocular coordination for both reflexive saccades and saccades belonging to reading sequences.

\section{TMS DISTURBED THE BINOCULAR COORDINATION OF READING FIXATIONS}

This study shows, for the first time, that the PPC is involved in the coordination of the eyes during post-saccadic fixation drift, whereas no such effect was found for fixations following single saccades (Vernet et al., 2008). The right PPC is involved in maintaining eye alignment during fixations, to keep disconjugate drift small, and for tailoring disconjugate drift appropriately to reduce misalignment caused by antecedent saccades.

Transcranial magnetic stimulation of the right PPC increased saccade and drift disconjugacy up to at least $600 \mathrm{~ms}$ after TMS pulse. Single-pulse stimulation is believed to deteriorate ongoing neural processes; perturbation could last up to $250 \mathrm{~ms}$ after stimulation (Jahanshahi and Rothwell, 2000). The perturbation up to $600 \mathrm{~ms}$ indicates that the right PPC is probably involved in regulation of binocular coordination of sequences of saccades lasting at least $600 \mathrm{~ms}$.

\section{RIGHT/LEFT PPC ASYMMETRY}

An important question is to what extend the effects of TMS are specific to the stimulated area, or due to an unspecific global effect related to stimulation. Several aspects are in favor of the first interpretation. The TMS effects were specific to the side of the PPC. TMS of the right PPC, but not left PPC, had an effect on binocular coordination of rightward reading saccades. Maybe with a larger group of subjects, increase of disconjugacy after left PPC stimulation might have reach significance. We are convinced that both right and left PPC might play a role in binocular control, with nevertheless a major implication of the right PPC. Indeed, such specialization of stimulation site is in line with the results of Vernet et al. (2008). Thus, it allows confidence on the specificity of the effects.

Similar functional dissociation between right and left PPC was previously found for the control of eye movement initiation. TMS over the right PPC increases the latency of all horizontal movements (Kapoula et al., 2001). TMS of the left PPC increases the latency of saccades to right, convergence, and convergence combined with rightward saccade (Yang and Kapoula, 2004). The left PPC would be specialized in coding the target location via its implication in triggering movements toward close and right space. The right PPC would have an omnidirectional and omnidepth role in fixation disengagement. All these studies support the idea that the right PPC is involved in several aspects of eye movement control (single saccades or reading saccades) while the left PPC has a more discreet involvement. 
Furthermore, for the first time, an implication of the right PPC in sequences of post-saccadic fixation disconjugacy was found. The right PPC is probably highly involved in eye movement control during reading, in line with fMRI observation by Grosbras et al. (2001), showing specific cortical activation in the right parietal area for learned sequences of saccades compared to new sequences. Thus, the control in 3D space, in particular the vergence angle control, would rely mostly on right PPC during reading.

\section{REGULATION OF INTRA-SACCADIC/POST-SACCADIC DISCONJUGACY}

Not only TMS increased saccade and fixation drift disconjugacy, but the relationship between these two parameters became looser: the drift disconjugacy was less efficient to reduce the misalignment caused by the saccade disconjugacy, probably resulting from a loss of sustained control mechanism of vergence.

Vergence deficit is often associated with high disconjugacy and a lack of correlation between saccade and drift disconjugacy, for example in children with dyslexia (for a review, see, Kapoula et al., 2008). Binocular coordination probably relies on vergence system. One possible origin of saccade disconjugacy is the existence of peripheral asymmetries, such as different visco-elastic properties between muscles of the eyes and different delay of innervation (Collewijn et al., 1988; Zee et al., 1992). We suggest that to compensate these asymmetries, the PPC learn to send a small vergence command together with every saccade. This vergence could go on after the end of the saccade, yet a pre-programmed vergence and a disparity-driven vergence could take place at the beginning of the fixation, causing a disconjugate drift that partly compensates for saccade disconjugacy. The PPC would be involved in these processes, which were disturbed by TMS.

As both perceptual and motor parameters are encoded in the PPC, one cannot exclude the possibility that the TMS disturbs the depth perception. The increase of fixation disconjugacy, which is believed to be, at least in its initial part, pre-programmed (Collewijn

\section{REFERENCES}

Blythe, H. I., Liversedge, S. P., Joseph, H. S., White, S. J., Findlay, J. M., and Rayner, K. (2006). The binocular coordination of eye movements during reading in children and adults. Vision Res. 46, 3898-3908.

Bucci, M. P., Bremond-Gignac, D., and Kapoula, Z. (2008). Poor binocular coordination of saccades in dyslexic children. Graefes Arch. Clin. Exp. Ophthalmol. 246, 417-428.

Bucci, M. P., Kapoula, Z., BremondGignac, D., and Wiener-Vacher, S. (2006). Binocular coordination of saccades in children with vertigo: dependency on the vergence state. Vision Res. 46, 3594-3602.

Bucci, M. P., Kapoula,Z., Yang, Q., Roussat, B., and Bremond-Gignac, D. (2002). Binocular coordination of saccades in children with strabismus before and after surgery. Invest. Ophthalmol. Vis. Sci. 43, 1040-1047.

Colby, L. C., Duhamel, J. R., and Goldberg, M. E. (1996). Visual, presaccadic, and

et al., 1988; Heller and Radach, 1999; Bucci et al., 2002), supports the existence of at least a motor component disturbed by stimulation of the PPC.

\section{CONCLUSION}

In conclusion, this study shows that binocular motor control during reading, although an automatic motor activity, involves complex $3 \mathrm{D}$ processes, aiming to keep saccade and drift disconjugacy small and to tailor drift disconjugacy appropriately to reduce misalignment of the eyes left at the end of the saccade. The right PPC is of major importance as its perturbation by single-pulse TMS disturb binocular coordination of both saccade and fixation. PPC is instrumental, acting online to keep the eyes aligned, but other cortical and subcortical structures (FEF, cerebellum) remain to be explored.

The data are of importance for understanding the origin of deficits of binocular coordination, frequently related to problems of vergence, e.g., children with dyslexia or vertigo (Bucci et al., 2006, 2008; Kapoula et al., 2009). The data are also of interest for models trying to identify cognitive and oculomotor mechanisms guiding eye movements during reading (see, e.g., Reilly and O'Regan, 1998). This study contributes in bridging the gap between oculomotor studies and reading studies. Indeed, in the last decade even cognitive models start to take into account physiological aspects of oculomotor control (Reichle et al., 1999; Engbert et al., 2002). The basic motor reading activity by itself deploys cortical resources and this should be included in future versions of models. We emphasized the role of the PPC, key structure during reading as it participates into both attention shifts and motor parameters.

Finally, one should point that global speed of reading remained unaffected even in the presence of local increase of disconjugacy. Although reading performance was not directly measured, the constancy of global speed is line with previous observation (Liversedge et al., 2006b) suggesting that reading and binocular fusion of the word is possible despite fixation disparities.

Oculomotor Research, ed. E. Becker (New York: Plenum Press).

Jahanshahi, M., and Rothwell, J. (2000). Transcranial magnetic stimulation studies of cognition: an emerging field. Exp. Brain Res. 131, 1-9.

Kapoula,Z., Isotalo, E., Muri, R. M., Bucci, M. P., and Rivaud-Pechoux, S. (2001). Effects of transcranial magnetic stimulation of the posterior parietal cortex on saccades and vergence. Neuroreport 12, 4041-4046.

Kapoula, Z., Vernet, M., Yang, Q., and Bucci, M. P. (2008). Binocular coordination of saccades: development, aging and cerebral substrate. J. Eye Mov. Res. 2, 1-20.

Kapoula, Z., Ganem, R., Poncet, S., Gintautas, D., Eggert, T., BremondGignac, D., and Bucci, M. P. (2009). Free exploration of painting uncovers particularly loose yoking of saccades in dyslexics. Dyslexia 15, 243-259.

Kapoula, Z., Yang, Q., Coubard, O., Daunys, G., and Orssaud, C. (2004). Transcranial magnetic stimulation of the posterior parietal cortex delays the latency of both isolated and combined vergence-saccade movements in humans. Neurosci. Lett. 360, 95-99.

Kapoula, Z., Yang, Q., Coubard, O., Daunys, G., and Orssaud, C. (2005). Role of the posterior parietal cortex in the initiation of saccades and vergence: right/left functional asymmetry. Ann. N. Y. Acad. Sci. 1039, 184-197.

Kirkby, J. A., Webster, L. A., Blythe, H. I., and Liversedge, S.P.(2008). Binocular coordination during reading and non-reading tasks. Psychol. Bull. 134, 742-763.

Leff, A. P., Scott, S. K., Rothwell, J. C., and Wise, R. J. (2001). The planning and guiding of reading saccades: a repetitive transcranial magnetic stimulation study. Cereb. Cortex 11, 918-923.

Leigh, R. J., and Zee, D. S. (2006). The Neurology of Eye Movements, 4th Edn. New York: Oxford University Press.

Liversedge, S. P., White, S. J., Findlay, J. M., and Rayner, K. (2006a). Binocular 
coordination of eye movements during reading. Vision Res. 46, 2363-2374.

Liversedge, S. P., Rayner, K., White, S. J., Findlay, J. M., and McSorley, E. (2006b). Binocular coordination of the eyes during reading. Curr. Biol. $16,1726-1729$.

Nuthmann, A., and Kliegl, R. (2009). An examination of binocular reading fixations based on sentence corpus data. J. Vis. 9, 1-28.

Reichle, E. D., Rayner, K., and Pollatsek, A. (1999). Eye movement control in reading: accounting for initial fixation locations and refixations within the E-Z Reader model. Vision Res. 39, 4403-4411.

Reilly, R. G., and O'Regan, J. K. (1998). Eye movement control during reading: a simulation of some wordtargeting strategies. Vision Res. 38, 303-317.

Sakata, H., Shibutani, H., and Kawano, K. (1980). Spatial properties of visual fixation neurons in posterior parietal association cortex of the monkey. J. Neurophysiol. 43, 1654-1672.

Stampe, D. M. (1993). Heuristic filtering and reliable calibration methods for video-based pupil-tracking systems. Behav. Res. Methods Instrum. Comput. 25, 137-142.

Vernet, M., and Kapoula, Z. (2009). Binocular motor coordination during saccades and fixations while reading: a magnitude and time analysis. J. Vis. $9,2$.

Vernet, M., Yang, Q., Daunys, G., Orssaud, C., Eggert, T., and Kapoula, Z. (2008).
How the brain obeys Hering's law: a TMS study of the posterior parietal cortex. Invest. Ophthalmol. Vis. Sci. 49, 230-237.

Yang, Q., and Kapoula, Z. (2003). Binocular coordination of saccades at far and at near in children and in adults. J. Vis. 3, 554-561.

Yang, Q., and Kapoula, Z. (2004). TMS over the left posterior parietal cortex prolongs latency of contralateral saccades and convergence. Invest. Ophthalmol. Vis. Sci. 45, 2231-2239.

Zee, D. S., Fitzgibbon, E. J., and Optican, L. M. (1992). Saccade-vergence interactions in humans. J. Neurophysiol. 68, 1624-1641.

Conflict of Interest Statement: The authors declare that the research was conducted in the absence of any commercial or financial relationships that could be construed as a potential conflict of interest.

Received: 31 July 2010; accepted: 24 January 2011; published online: 07 February 2011. Citation: Vernet M, Yang $Q$ and Kapoula $Z$ (2011) Guiding binocular saccades during reading: a TMS study of the PPC. Front. Hum. Neurosci. 5:14. doi: 10.3389/ fnhum.2011.00014

Copyright (c) 2011 Vernet, Yang and Kapoula. This is an open-access article subject to an exclusive license agreement between the authors and Frontiers Media $S A$, which permits unrestricted use, distribution, and reproduction in any medium, provided the original authors and source are credited. 